\title{
Rotational kinetic therapy for ventilation/perfusion mismatch
}

\author{
Barbara McLean, MN, RN, CCRN, CRNP, CCNS, FCCM, Nurse Intensivist, Atlanta Medical Center and \\ Grady Hospital, Atlanta, GA, USA
}

Atlanta Medical Center and Grady Hospital, 1444 Cornell Road Atlanta GA 30306. e-mail: bamclean@mindspring.com

\section{INTRODUCTION}

One of the most significant and serious challenges of modern critical care practice is systemic inflammatory response syndrome (SIRS). It is often heralded by acute pulmonary parenchymal changes causing refractory hypoxaemia (Table 1). The process is generally classified, according to the degree of alveolar dysfunction and atelectasis, as either acute lung injury (ALI) or acute respiratory distress syndrome (ARDS).

The nidus for injury may be direct (for example, from inhalation or inspiration of noxious substances) or indirect (from an overwhelming inflammatory mediation, which is proteindenaturing in nature). However, the result remains the same. The patient experiences a failure to oxygenate despite an intake of high-percentage oxygen.

ALI is a common diagnosis for critical patients in the ICU. Recently, the overall hospital mortality rate reported for patients diagnosed with ALI exceeded 50\% (Hartog et al., 1997). Some studies have indicated that significant lung density in these conditions is a result of atelectasis rather than focal injury alone (Hartog et al., 1997; Deb and Pearl, 1997). Although there may be structural changes associated with inflammatory or injurious processes, the corresponding atelectasis may indeed cause proliferative hypoxaemia.

The therapeutic challenge in the management of ALI and ARDS is the promotion of adequate gas exchange. Despite an $\mathrm{FiO}_{2}$ of 0.70 or greater, the arterial blood gas reflects an inadequate exchange relationship between alveolus and arterial blood.

One of the major differentiating factors in ALI and ARDS is the degree of refractory hypoxaemia. Due to profound atelectasis, alveolar collapse and ductal closure, hypoxaemia will

\section{Table 1. Evaluation of ALI and ARDS}

Presence of refractory hypoxaemia

$-\mathrm{PaO}_{2} / \mathrm{FiO}_{2}(\mathrm{P} / \mathrm{F})<300$ on greater than $0.40 \mathrm{FiO}_{2}$ : acute lung injury

$-\mathrm{PaO}_{2} / \mathrm{FiO}_{2}(\mathrm{P} / \mathrm{F})<200$ on greater than $0.40 \mathrm{FiO}_{2}$ : acute respiratory distress syndrome

- Intrapulmonary shunt and ventilation/perfusion mismatch (V/Q mismatch)

- Increased pulmonary vascular permeability with primarily dependent infiltrates seen on chest X-ray

- Decreased lung compliance and decreased functional residual capacity (peak inspiratory pressure $>45 \mathrm{~cm} \mathrm{H} \mathrm{H}_{2} \mathrm{O}$ (Verbrugge et al., 1997; Cawley et al., 1998; Sandiford et al., 1995; Sinclair et al., 1997)

- Dependent lung atelectasis worsen if intervention is not swift and directed towards ductal opening and alveolar recruitment, as well as redistribution of distending airway pressures. These patients may be profoundly haemodynamically unstable; they will need invasive monitoring and frequent arterial blood gas measurements, particularly for the presence of metabolic acidosis. The patient profile in early ALI or ARDS will often present with acute pulmonary hypertension.

As the patient becomes more ischaemic and hypoxic, the haemodynamic and therapeutic interventions may require that the patient is aggressively sedated and even paralysed. The patient may not be moving and may depend upon the nursing staff for implementation of turning protocols. In this situation, the dependent, dorsal aspects of their lungs may become significantly under-ventilated, flooded and atelectatic. Factors that may predispose or exacerbate the more serious conditions known as ALI and ARDS include:

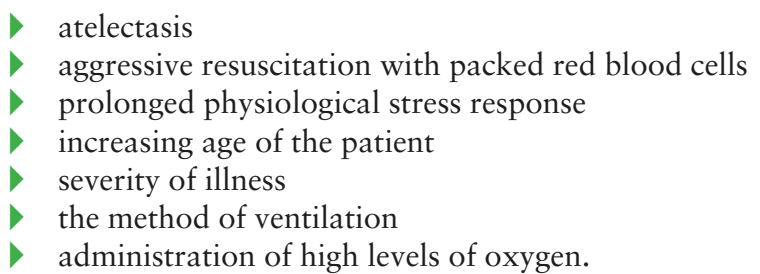

Although the patient may have structural changes associated with inflammatory or injurious processes, loss of surfactant protection and failure of sodium $\left(\mathrm{Na}^{+}\right)$and $\mathrm{H}_{2} \mathrm{O}$ pumps, the corresponding atelectasis may actually be the evolving cause of proliferative hypoxaemia. The traditional critical care approach has been to increase $\mathrm{FiO}_{2}$ and positive end-expiratory pressures (PEEP).

\section{VENTILATION}

Normal alveolar ventilation ranges around 4 litres/minute (West, 1990). The West model of the lung (Figure 1) demonstrates the normal gas and blood match. As gas is delivered through active contraction of the diaphragm, creating negative pressure in the thoracic cage, the majority of gas is delivered to the independent (i.e. uppermost) lung.

In order for effective ventilation to occur, the patient must have: 


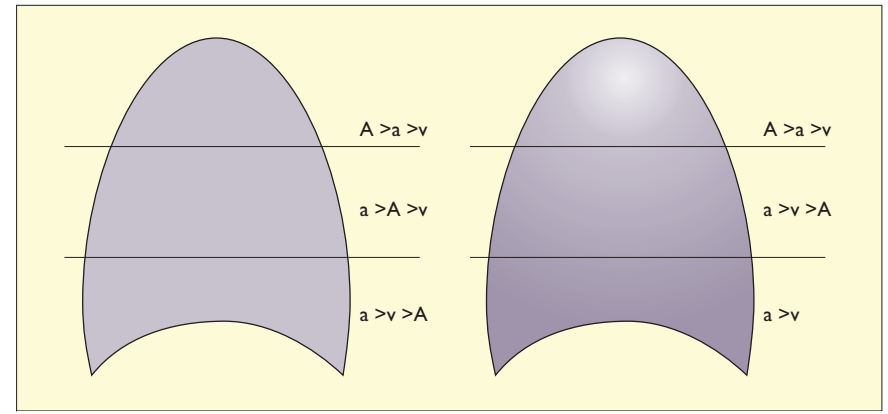

Figure 1. The West model for ventilation perfusion match in the normal (left) and abnormal (right) lung. The relationship portrayed shows the blood-gas relationship.

intact innervation of inspiratory muscle groups

negative intrapleural pressure

- low resistance in the conducting airways

- open alveolar ducts

- alveolar compliance.

In order for effective perfusion to occur, the patient must have:

- an adequate volume to fill the ventricles

the ventricles must be compliant (venous filling, i.e. preload)

- the ventricles must be able to generate tension against resistance of the two arterial beds (pulmonary and systemic, i.e. afterload)

- the contractile apparatus (ejection: stoke volume or cardiac index) of both ventricles must be relatively intact.

Ventilation $(\mathrm{V})$ to perfusion $(\mathrm{Q})$ is maximised by the mobilisation of air to the dependent lung surface by negative pressure (normal breathing) and the perfusion of the dependent lung by the higher pressure and volume of blood flow (Figure 1). The delicate balance of ventilation to perfusion is maximised in a patient with a significant negative inspiratory force and a normal cardiac index. In critical illness, when hypoventilation is common, positive pressure ventilation is the choice of treatment and perfusion may be significantly altered. Thus, it is easy to see why ventilation and perfusion may be mismatched.

When ventilation is greater than perfusion, the term 'dead space' is used. That is, if alveoli are distended and ventilated, but blood flow past the alveoli is decreased, the oxygenation levels of the blood will drop. This condition, known as high V to Q, exists when the patient suffers a pulmonary embolism or has a profound decrease in cardiac stroke volumes.

When perfusion is greater than ventilation, the term 'intrapulmonary shunt' is used. In true shunt, alveolar collapse due to lung injury and/or atelectasis significantly affects the actual lung surface available for gas exchange. The compensatory response to the decrease in oxygen is to increase the heart rate and cardiac stroke volume. So just as ventilation is going down, perfusion (if the cardiac function is normal) is rising.

Blood is heavier than gas and always is maximised in the dependent area. This perfusion $(\mathrm{Q})$ is measured at about 5 litres/ minute. In the zone where blood and gas meet, but the pressure of blood is higher than the pressure of air, is the normal V/Q match of 0.8 litres/minute.

The disorders that inhibit ventilation are classified as either obstructive or resistant. Obstructive airway disease is typically a dysfunction of air trapping in the alveoli, increasing alveolar compliance and the content of alveolar $\mathrm{CO}_{2}$ and decreasing the content of alveolar $\mathrm{O}_{2}$. Resistant airway disease occurs when there is compression of the alveolar sacs, such as in pneumothorax, or when there is a loss of alveolar compliance, as in ARDS and atelectasis.

Some of the mechanisms involved in ALI and ARDS are destruction of the alveolar membrane, the surfactant producing cells, presence on extravascular lung water and failure of the sodium- $\mathrm{H}_{2} \mathrm{O}$ pump. This promotes alveolar sac collapse and loss of functional residual capacity. The loss of functional lung surface is often referred to as shock lung or baby lung, that is, the adult lung may only have a small recruitable surface available for gas exchange. Delivery and retention of positive pressure gas to a small surface lung, one that has significant loss of recruited alveolar compartment structures may also result in significant volume or pressure trauma.

The process of atelectasis, alveolar flooding, and collapse may require therapeutic strategies designed to recruit functional, although atelectatic, alveoli by air trapping and increasing endexpiratory pressures. The methods used for this purpose (inverse ratio ventilation, permissive hypercapnoea, high-frequency ventilation), provide either mechanical PEEP or circuit endexpiratory pressures (Burchardi, 1996; Zavala et al., 1998).

With PEEP, the majority of gas will be delivered to the independent surface of the lung, while the blood flow tends towards the dependent surface. There is a portion of gas that never meets blood (alveolar dead space) and a portion of blood that never meets gas (intrapulmonary shunt). Depending on the pathology involved, this mismatch of gas and blood (V/Q mismatch) may be heightened to the point that the patient presents with a low arterial oxygen: inspired oxygen ratio $(\mathrm{P} / \mathrm{F})$ of less than 200, and a wide alveolar oxygen-arterial oxygen (A-a) difference of greater than $100 \mathrm{~mm} \mathrm{Hg}$.

The delivery of volumes greater than $8 \mathrm{ml} / \mathrm{kg}$ or the use of distending pressures greater than $10 \mathrm{~cm} \mathrm{H}_{2} 0$, will primarily be delivered to the independent lung surface. This will encourage over-distension of the independent lung surface, and may cause barotrauma and further exacerbation of alveolar dysfunction. The application of tidal volume and positive pressure (circuit or mechanical) should be distributed throughout the entire lung surface if the desired effect is to recruit alveoli for gas exchange.

These ventilation strategies can be very costly. So far, the literature does not fully support the advantages of such therapies. In fact, some types of ventilatory support may significantly increase the shunt, V/Q mismatch and alveolar destruction in critical patients (Figures 1 and 2).

\section{POSITIONING}

Position change has been suggested as a relatively inexpensive intervention that promotes redistribution of gas, recruitment of alveoli, redistribution of blood flow and may even stimulate atrial natriuretic hormone release (Servillo et al., 1997). Despite the many studies reinforcing the effect of positional changes on

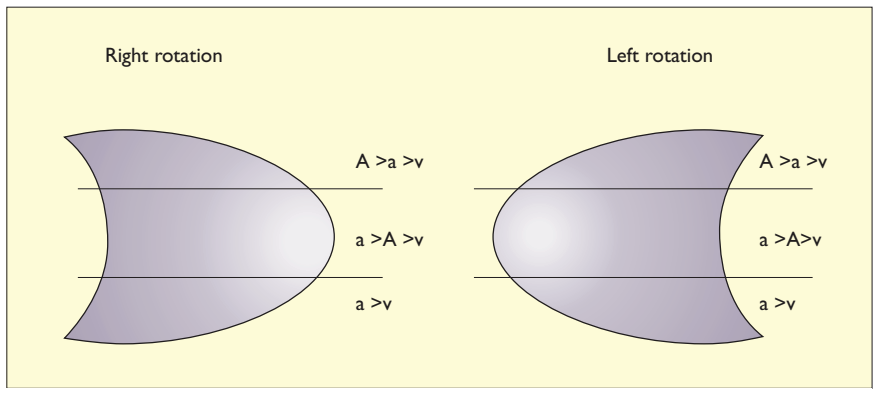

Figure 2. The proposed effect of steep lateral rotation on the blood-gas relationship. 
the incidence of pneumonia in the critically ill patient, and studies validating the role of prone positioning in the patient with ARDS (Deb and Pearl, 1997; Stocker et al., 1997), there has been little attention paid to the preventative effects of positional change in the patient at risk for ALI and ARDS.

Since positional changes have been well supported as a method of increasing V/Q match, the early use of kinetic therapy $\left(40^{\circ}\right.$ or greater rotation to each side) may prevent ALI, accumulation of inflammatory infiltrates, and concomitant atelectasis (Deb and Pearl, 1997; Hartog et al. 1997).

Continuous kinetic therapy as an early preventive tool for ALI and ARDS has not been evaluated, most probably because it is difficult to predict which patients will develop ALI.

The use of kinetic treatment meets both research and commonsense criteria. Multiple studies have shown conclusive evidence that aggressive position changes redistribute exudate, alleviate pressure and increase alveolar recruitment. From the commonsense perspective, with positive pressure ventilation, gas is primarily moved to the independent lung surface. This may be validated by lung computerised tomography (CT) scan, thus demonstrating that no lung should be kept dependent for any length of time (althougth there is no consensus on the amount of time the lung may be dependent). There are other benefits associated with kinetic positioning. Some of the reasons for lung protection may include, but are not limited to, those given in Table 2.

Some of the factors that limit early, aggressive and committed kinetic therapy are related to the patient's condition. The patient must rotate in a full $80-90^{\circ}$ arc for about 18 hours each day. This treatment regimen relies on an organised work plan, which limits rotation only when necessary.

As patients rotate, they may become tachycardic and hypotensive and they may desaturate. These are common issues and may be addressed relatively simply. As patients turn, the blood flows towards the most dependent area, and the patient may suffer from some form of orthostatic hypotension. Volume resuscitation is essential in this case. Patients typically desaturate when their 'bad' lung is down, so the time for this position may need to be decreased for the first 24 hours, but should not be withheld if at all possible.

Other problems may be best addressed with acclimation to rotation. Acclimation allows achievement of a maximal turn over a period of time, similar to increasing the $G$ force on an astronaut in training.

\section{STUDY HYPOTHESIS}

We hypothesised that aggressive kinetic rotation would decrease the incidence of refractory hypoxaemia in a group of patients matched by injury with documented risk factors for ALI or ARDS, including injury severity, enforced immobility, mechanical ventilation and volume and packed red blood cell resuscitation.

Table 2. Potential benefits of lung protection
Benefits
Recruitment of alveoli
Equalised distribution of airway volume and pressures
Redistribution of inflammatory fluid
Increased lymphatic pressure, promoting drainage
Increased capillary blood flow with decreased inflammatory stimulation
Increased lung compliance
Increased atrial natriuretic peptide hormone release

\section{Methodology}

This study was conducted in the trauma-surgical intensive care unit of a university-affiliated, level one, trauma center. The institutional review boards of the Emory School of Medicine, the Morehouse School of Medicine and the Grady Hospital evaluated and approved the proposal for scientific and ethical content.

Over an 18-month period, 45 patients with blunt or penetrating injuries and between the ages of 17 and 65 years of age met the enrolment criteria. Inclusion criteria dictated that patients have:

- an injury severity score (ISS) (Osler, 1996) of $\geq 16$

- were mechanically ventilated with $\mathrm{PaO}_{2} / \mathrm{FiO}_{2}(\mathrm{P} / \mathrm{F})$ ratio $<225$ (as many patients in our ICU have $\mathrm{P} / \mathrm{F}<300$ initially and $<200$ was considered to low)

- a family member was available to give informed consent.

An injury value of 4 was given for an estimated blood loss $\geq 20 \%$ of the patient's circulating volume.

Patient enrolment in the study had to occur within 24 hours following a traumatic injury. Patients were managed by the oncall trauma residents, fellows, and attending physicians of two different medical school programmes, with some differences in therapeutic approach. (The difference of approaches related primarily to choice of initial ventilation (i.e. SIMV or CMV), and did not appear to effect the outcomes of the study. Two patients were enrolled from one medical school, the rest from the other.

At the time of entry, the patients' arterial blood gases, pulse oximetry, chest radiograph and vital signs were evaluated. Patients with uncleared or documented cervical spinal cord injury, intracranial pressures $>20 \mathrm{~mm} \mathrm{Hg}$ or a Glasgow Coma score of 8 at 24 hours were excluded from the study. After informed consent had been obtained and after evaluating entry data, patients were prospectively randomised into one of two groups:

- Control group (conventional treatment); turning of patient according to the unit protocol (every 2 hours using wedge or pillows).

- Experimental group (kinetic); placement on a kinetic therapy surface (TriaDyne ${ }^{\circledR}$, Kinetic Concepts Inc., San Antonio, Texas, USA).

Turning protocols for the experimental group required that the patient achieve a maximal rotation angle of $45^{\circ}$ per side for a total arc of $90^{\circ}$ within 4 hours of placement. Rotation was to occur for 18 hours per day at a total $90^{\circ}$ rotation arc. Patients achieved maximal rotation through an acclimation period that increased degree achievement over 2 hours. Patients would pause for 10 minutes to the left, then 5 minutes supine, and 10 minutes to the right. These pause times were chosen to allow for recruitment of the alveoli and even gas distribution to the lung.

All nursing procedures were performed during short rest periods from rotation. Both mechanical and manual rotation occurred during both day and night hours. Neither group received chest physiotherapy or percussion during their duration of stay in the study. All patients received volume, inotropic and vasoactive support as necessary. No patients received direct pulmonary vasodilators.

Daily data was collected on all patients regarding ISS, ventilator settings, arterial blood gases and calculated P/F. Study points were measured every morning at four o'clock following 4 hours of uninterrupted rotation. Daily chest radiographs were 
interpreted according to the physician standards of the radiology department. In addition, arterial blood gases, vital signs and haemodynamic measurements were evaluated, as dictated by the patient's condition and unit protocols.

\section{Results (Tables 4 to 8)}

During the period in ICU, eight $(40 \%)$ patients in the control group died. The diagnosed causes of death were severity of injury, exsanguination, myocardial infarction, ARDS and associated organ failure. There were no deaths in the experimental group. However, five patients in the experimental group were unable to complete their rotation requirements, due to frequent operating room visits, profound haemodynamic instability, and an inappropriate decrease in rotation. Patients were not followed-up after discharge from the intensive care unit or the hospital.

All patients enrolled in this study were prospectively randomized into the two study groups: conventional or kinetic therapy. There was no significant difference between the profiles of the two groups in respect to age, sex or incidence of abdominal injury (Tables 3, 4, 5). Estimated blood loss ranged from 200 to $10,000 \mathrm{ml}$ with seven patients unreported. The mean admission P/F, base excess (BE) and ISS of the two groups were similar (Table 3). Forty-one patients (91\%) suffered from penetrating injuries and four $(9 \%)$ from blunt injury. Ten

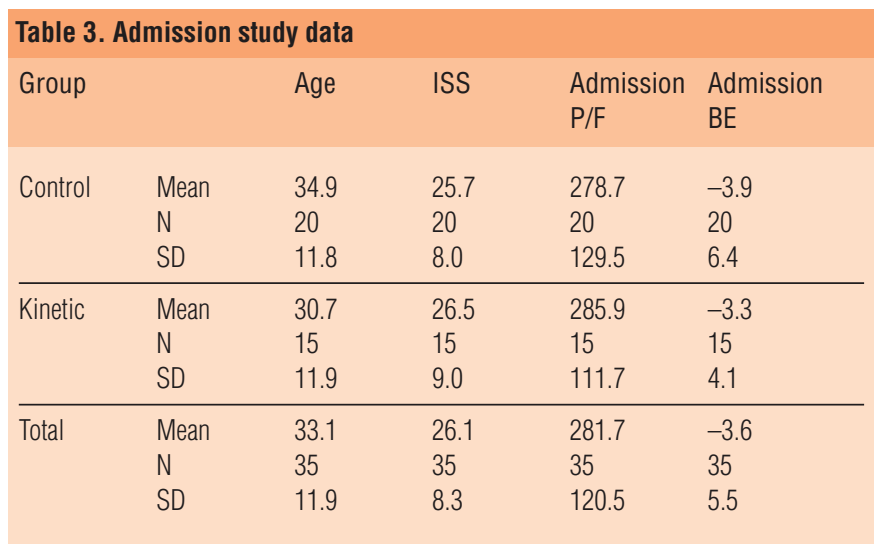

\begin{tabular}{|c|c|c|c|c|c|}
\hline & & & $\begin{array}{l}\text { Gender } \\
\text { F }\end{array}$ & $\mathrm{M}$ & Total \\
\hline \multirow[t]{2}{*}{ Group } & Control & $\begin{array}{l}\text { Count } \\
\% \text { within group }\end{array}$ & $\begin{array}{l}3 \\
15.0 \%\end{array}$ & $\begin{array}{l}17 \\
85.0 \%\end{array}$ & $\begin{array}{l}20 \\
100 \%\end{array}$ \\
\hline & Kinetic & $\begin{array}{l}\text { Count } \\
\% \text { within group }\end{array}$ & $\begin{array}{l}1 \\
6.7 \%\end{array}$ & $\begin{array}{l}14 \\
93.3 \%\end{array}$ & $\begin{array}{l}15 \\
100 \%\end{array}$ \\
\hline Total & & $\begin{array}{l}\text { Count } \\
\% \text { within group }\end{array}$ & $\begin{array}{l}4 \\
11.4 \%\end{array}$ & $\begin{array}{l}31 \\
86.6 \%\end{array}$ & $\begin{array}{l}35 \\
100 \%\end{array}$ \\
\hline
\end{tabular}

\begin{tabular}{|c|c|c|c|c|c|}
\hline & & & \multicolumn{2}{|c|}{ Abdominal injury } & \multirow[t]{2}{*}{ Total } \\
\hline & & & & Yes & \\
\hline \multirow[t]{4}{*}{ Group } & Control & Count & 6 & 14 & \\
\hline & & $\%$ within group & $30.0 \%$ & $70.0 \%$ & $100 \%$ \\
\hline & Kinetic & Count & 3 & 12 & 15 \\
\hline & & $\%$ within group & $20.0 \%$ & $80.0 \%$ & $100 \%$ \\
\hline \multirow[t]{2}{*}{ Total } & & Count & 9 & 26 & 35 \\
\hline & & $\%$ within group & $25.7 \%$ & $74.3 \%$ & $100.0 \%$ \\
\hline
\end{tabular}

patients did not complete the study: three died in the first 10 hours after enrolment; two self-extubated, and five did not complete rotation requirements due to frequent operating room visits or profound refractory haemodynamic instability.

Thirty-five patients completed the study protocol. In the control group, 15 patients $(75 \%)$ experienced increased ventilator changes that signified difficulty with oxygenation and/or ventilation (Table 6). Seven patients $(35 \%)$ in the control group required increased $\mathrm{FiO}_{2}$, frequency, or tidal volume. Eight $(40 \%)$ patients in the conventional group required either pressure control inverse ratio or high frequency jet ventilation to maintain minimal oxygenation. One patient $(6.7 \%)$ in the kinetic therapy group required an increase in $\mathrm{FiO}_{2}$. Utilizing standard criteria for evaluation of ALI and ARDS, 10 patients $(50 \%)$ in control group were positively diagnosed (Table 7). These patients required increased ventilator support as referenced above, that is, they required a significant increase in support. In addition they had three consecutive P/F ratios of less than 225 and exacerbation of infiltrates on chest radiograph. No patients in the experimental group who achieved daily rotation requirements displayed the diagnostic indicators for ALI/ARDS.

During the intensive care unit stay, eight $(40 \%)$ patients in the control group died. Severity of injury, exsanguination, myocardial infarction, ARDS and associated organ failure were the diagnosed causes of death. No patients in the experimental group expired, however, some patients were unable to complete their rotation requirements due to frequent operating room visits, profound haemodynamic instability, and inappropriate decrease in rotation (Table 8 ).

In conclusion, our results suggest that aggressive rotation has a positive impact on the lung function of severely injured trauma patients at risk for atelectasis, ALI and ARDS.

\section{DISCUSSION}

This pilot study found that in a similar group of individuals who had multiple risk factors for ALI and ARDS, progressive lateral rotation or Kinetic Therapy $\left(45^{\circ}\right.$ or total $90^{\circ}$ arc, 10-5-10minute pause time in rotation, 18 hours per day) provided a

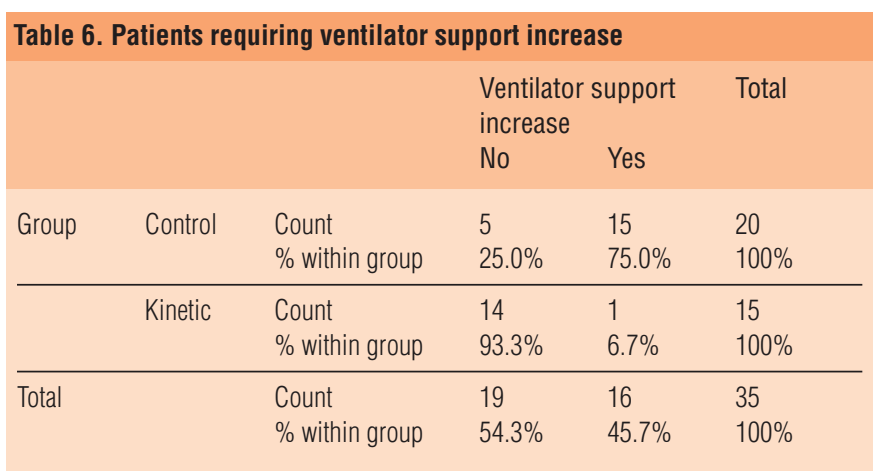

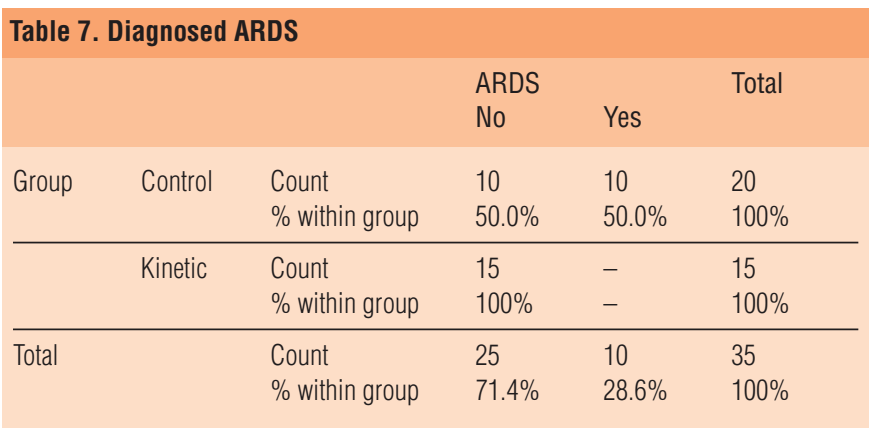




\begin{tabular}{|c|c|c|c|c|c|}
\hline & & & \multicolumn{2}{|c|}{ Patient died } & \multirow[t]{2}{*}{ Total } \\
\hline & & & No & Yes & \\
\hline \multirow[t]{2}{*}{ Group } & Control & $\begin{array}{l}\text { Count } \\
\% \text { within group }\end{array}$ & $\begin{array}{l}12 \\
60.0 \%\end{array}$ & $\begin{array}{l}8 \\
40.0 \%\end{array}$ & $\begin{array}{l}20 \\
100 \%\end{array}$ \\
\hline & Kinetic & $\begin{array}{l}\text { Count } \\
\% \text { within group }\end{array}$ & $\begin{array}{l}15 \\
100 \%\end{array}$ & $\begin{array}{l}- \\
-\end{array}$ & $\begin{array}{l}15 \\
100 \%\end{array}$ \\
\hline Total & & $\begin{array}{l}\text { Count } \\
\% \text { within group }\end{array}$ & $\begin{array}{l}27 \\
77.1 \%\end{array}$ & $\begin{array}{l}8 \\
22.9 \%\end{array}$ & $\begin{array}{l}35 \\
100 \%\end{array}$ \\
\hline
\end{tabular}

protective mechanism for the lung. In conjunction with protective lung ventilation and increasing pulmonary perfusion, redistribution of gas and blood in the lung may significantly alter the patient's progress. It is vital to promote the theory that no lung surface should be dependent for any period of time, in fact if lung surfaces are maintained in a primarily dependent position, atelectasis, exudative accumulation and lung failure may progress to mortality.

Our results suggest that aggressive rotation may have a positive impact on the lung function of severely injured trauma patients at risk for atelectasis, ALI and ARDS.

In a similar group of individuals, with multiple risk factors for ALI and ARDS, progressive lateral rotation or kinetic therapy may provide a protective mechanism for the lung.

In assessing the results of the study, certain limitations should be taken into account. These patients were relatively young with few or no co-morbid conditions confounding their injury. Progressive rotation was introduced early in order to promote equal bilateral gas distribution to the lung and therefore significantly reducing the risk of atelectasis. The continued maintenance of aggressive kinetic therapy required a significant commitment from the surgical intensive care unit's nursing staff and was achieved with very intensive education and support. Haemodynamic values were not tracked in this patient population; severe haemodynamic instability was treated with volume, inotropes and vasoconstrictors. Haemodynamic changes associated with rotation were not considered a reason to discontinue therapy, but these changes were often seen.

The aggressive application of rotation has not been universally tested. For our purposes, kinetic rotation was considered to be a rotation arc of $90^{\circ}$, with a 10 -minute/5-minute/10-minute pause, 18 hours per day. The angle of rotation of $90^{\circ}$ is generally accepted. However, the time allowed for pausing and the amount of hours required for therapy have not yet been defined.

In order to achieve the maximal rotation defined in this study, patients required continuous intravenous sedation, which may have been a limiting factor for some patients. The sedation required for the study patients was not equally matched in the control population.

Patient comfort, desaturation and haemodynamic variability may limit the bedside nurse's desire to rotate the patient aggressively, However, with a renewed commitment to long-term outcome, it is essential to plan for some level of desaturation (acceptability defined by the context, i.e. lactic acidaemia) and haemodynamic changes.

\section{ACKNOWLEDGEMENTS}

The author would like to express appreciation to Trauma Directors, Dr Grace Rozycki and Dr Karyn Butler, SICU Clinical Manager, Carolyn Steed, Trauma Nurse, Valerie Barnes, and the residents and nursing staff of the SICU at Grady Memorial Hospital, Atlanta, Georgia, USA.

\section{REFERENCES}

Bein T, Reber A, Metz C, Jauch KW, Hedenstierna G. (1998). Acute effects of continuous rotational therapy on ventilation-perfusion inequality in lung injury. Intensive Care Medicine; 24: 132-137.

Burchardi H. New strategies in mechanical ventilation for acute lung injury. European Respiratory Journal; 1996; 9: 1063-1072.

Cawley MJ, Skaar DJ, Anderson HL 3rd, Hanson CW 3rd. (1998). Mechanical ventilation and pharmacologic strategies for acute respiratory distress syndrome. Pharmacotherapy; 18: 140-155.

Deb B, Pearl RG. (1997). Mechanical ventilation and adjuncts in acute respiratory distress syndrome. International Anesthesiology Clinics; 35: 109-124.

Hartog A, Houmes RJ, Verbrugge SJ, Erdmann W, Lachmann B. (1997). Partial liquid ventilation and inhaled nitric oxide have a cumulative effect in improving arterial oxygenation in experimental ARDS. Advances in Experimental Medicine and Biology; 428: 281-283.

Osler T, Rutledge R, Dies J et al. (1996). The ICD-9-based illness severity score: a new model that outperforms both DRG and APR-DRG as predictors of survival and resource utilization. Journal of Trauma; 45: 791-799.

Sandiford P, Province MA, Schuster DP. (1995). Distribution of regional density and vascular permeability in the Adult Respiratory Distress Syndrome. American Journal of Respiratory Critical Care Medicine; 151: 737-742.

Servillo G, Roupie E, De Robertis E, Rossano F, Brochard L, Lemaire F, Tufano R. (1997). Effects of ventilation in ventral decubitus position on respiratory mechanics in adult respiratory distress syndrome. Intensive Care Medicine; 23: 1219-1224.

Sinclair SE, Albert RK. (1997). Altering ventilation-perfusion relationships in ventilated patients with acute lung injury. Intensive Care Medicine; 23: 942-950.

Stocker R, Neff T, Stein S, Ecknauer E, Trentz O, Russi E. (1997). Prone positioning and low-volume pressure-limited ventilation improves survival in patients with severe ARDS. Chest; 111: 1008-1117.

Verbrugge SJ, Sorm V, Lachmann B. (1997). Mechanisms of acute respiratory distress syndrome: role of surfactant changes and mechanical ventilation. Journal of Physiology and Pharmacology; 48: 537-557.

West, JB. (1990). Respiratory Physiology, the essentials. Baltimore, MD: Williams and Wilkins.

Zavala E, Ferrer M, Polese G, Masclans JR, Planas M, Milic-Emili J, Rodriguez Roisin R, Roca J, Rossi A. (1998). Effect of inverse I:E ratio ventilation on pulmonary gas exchange in acute respiratory distress syndrome. Anesthesiology; 88: $35-42$. 